\title{
Species composition, diversity and traditional uses of plants in homegardens in Kampung Masjid Ijok, Perak, Malaysia
}

\author{
MOHD RAZNAN RAMLI ${ }^{1, \boldsymbol{\nu}}$, POZI MILOW ${ }^{1}$, SORAYYA MALEK ${ }^{2}$ \\ ${ }^{1}$ Department of Environmental Management, Faculty of Science, Institute of Biological Sciences, University of Malaya. 50603 Kuala Lumpur, Malaysia. \\ Tel.: +60-3-79676777, `email: raznan83@ gmail.com \\ ${ }^{2}$ Department of Bioinformatics Management, Faculty of Science, Institute of Biological Sciences, University of Malaya. 50603 Kuala Lumpur, Malaysia
}

Manuscript received: 24 February 2021. Revision accepted: 23 March 2021.

\begin{abstract}
Ramli MR, Milow P, Malek S. 2021. Species composition, diversity and traditional uses of plants in homegardens in Kampung Masjid Ijok, Perak, Malaysia. Biodiversitas 22: 1902-1911. The aim of present study was to analyze the status, plant diversity and their utilization in homegardens of Kampung Masjid Ijok, Perak, Malaysia. Forty homegardens were selected for detailed study: 9 were large $\left(>1,500 \mathrm{~m}^{2}\right), 11$ medium $\left(901 \mathrm{~m}^{2}\right.$ to $\left.1500 \mathrm{~m}^{2}\right)$ and $20 \mathrm{small}\left(<900 \mathrm{~m}^{2}\right)$. A total of 207 plant species belonging to 78 families were recorded. Among the usable components, food plants dominated the homegardens with a proportion of $43.3 \%$ followed by ornamental $(27.8 \%)$ and medicinal (24\%) plants. Large homegardens were dominated by food, medicinal and ornamental plant species, medium homegardens food and medicinal plant species, and small homegardens ornamental plant species. Frequency of species occurrences increased with the increasing homegardens size. The highest Shannon-Weiner Index was recorded in the large sized homegardens (3.61) and the lowest one in the small-sized homegardens (1.66). Euphorbiaceae (13 species), Araceae (11 species) and Fabaceae (10 species) were the most represented plant families. Shrubs dominated the plants in all homegardens with a proportion of $42 \%$, followed by trees (29.5\%), herbs (23.7\%) and climbers (4.8\%). Musa paradisiaca L. was recorded as a very frequent species, followed by Cocos nucifera L., Nephelium lappaceum L., Capsicum frutescens L., Carica papaya L, Cymbopogon citratus (DC.) Stapf, Mangifera indica L, Curcuma domestica Valeton, Garcinia mangostana L and Manihot esculenta Crantz. Alocasia sanderiana and Aquilaria malaccensis categorized as critically endangered (CR) in (IUCN) Red List were also recorded in the large homegardens. This study suggested that large homegardens are more effective than the small and medium ones for diversification and conservation of plant species.
\end{abstract}

Keywords: Homegardens, Kampung Masjid Ijok, Perak, plants diversity

\section{INTRODUCTION}

Homegardens are less complex agroforests almost similar to natural forest ecosystems but are integrated into agricultural management systems located around houses (Mapongmetsem et al. 2012). According to Bargali et al. (2019), homegarden is a traditional land use practice around a homestead where members of the household maintain several plant species and their products are intended primarily for household consumption. There are many plant species in the homegardens to grow foodbearing plants for household use (Galhena et al. 2013). According to Mattsson et al. (2017), homegardens can provide a stable source of fresh and nutritious food. Some plants have medicinal values that are a less expensive healthcare source and treatment for various illnesses and diseases. Panyadee et al. (2019) recorded 95 medicinal plants in homegardens of indigenous community in Thailand. Infection and infestation, nutritional disorders, and digestive system disorders were the three most important medicinal use categories for plants in the homegardens (Panyadee et al. 2019). In Eastern Himalaya, 53 plant species were utilized for different ethnomedicinal applications (Pala et al. 2019). In addition, the importance of homegardens for the conservation of plant species has also been reported in the literature (Ebert 2020). According to Bardhan et al. (2012), homegardens have the potential to increase biodiversity in the agricultural landscape while reducing habitat loss and fragmentation. Gachuiri et al. (2017) also indicated that homegardens play an important role in biodiversity conservation while sustaining livelihood.

Some homegardens products are sold as an additional income (Avilez-Lopez et al. 2020). Study by Abdoellah et al. (2020) in West Java, Indonesia indicated that homegardens are highly commercialized, which contributes to the spread of monocultural production in the region. Plants in homegardens are arranged in different configurations and vertically organized in a multi-layered structure (George and Christopher 2020) which resembles neighboring forest ecosystems (Mattsson et al. 2013) and thus provides multiple environmental benefits. The ability of homegardens to store carbon and enhance carbon sequestration is critical for climate change mitigation (Mattsson et al. 2015). Study in Moneragala District, Sri Lanka indicated that homegardens stored 13 Mega grams of carbon per hectare $\left(\mathrm{Mg} \mathrm{Cha}^{-1}\right)$ with a large range among homegardens ( 1 to $56 \mathrm{Mg} \mathrm{C}^{-1}$ ) due to a variety of tree diversity and composition between individual homegardens. Homegardens are also the perfect habitat for certain insects and pollinators (Liu et al. 2018) which provides essential support for food security and ecosystem stability (Lautenbach et al. 2012). The ability of homegardens to preserve soil structure and prevent soil 
erosion (Linger 2014), cycling soil nutrients (Reetsch et al. 2020) and preserving soil organic matter (Mohri et al. 2013) has been documented in the literature. In Malaysia, the research on homegardens is still scanty. Among the studies that had been conducted was by Milow et al. (2013). In this study, 214 plant species and varieties belonging to 76 families were recorded from the 10 Orang Asli villages studied in Negeri Sembilan, Malaysia (Milow et al. 2013). In view of the significant contribution and benefits of homegardens to human well-being and the inadequate exploration of homegardens in Malaysia, this study focuses on providing useful information that can support and encourage local communities to develop their homegardens in a sustainable manner.

\section{MATERIALS AND METHODS}

The study was conducted in the year 2018 in a village known as Kampung Masjid Ijok which lies between $5^{0} 7^{\prime} 0^{\prime \prime} \mathrm{N}$ to $5^{0} 8^{\prime} 15^{\prime \prime}$ latitude and $100^{\circ} 49^{\prime} 15^{\prime \prime} \mathrm{E}$ to $100^{\circ} 50^{\prime} 45^{\prime \prime} \mathrm{E}$ longitude in Selama Municipality, in the State of Perak, Malaysia (Figure 1). The village lies approximately 50 meters above sea level and extends $35.974 \mathrm{~km}^{2}$ and only comprises Malay community. It has a tropical climate with temperatures recorded from $28^{\circ} \mathrm{C}$ to $32^{\circ} \mathrm{C}$ through the whole year and $2,000 \mathrm{~mm}$ to $2,500 \mathrm{~mm}$ of precipitation annually. Land cover includes rubber tree and palm oil plantations and natural forests. The population comprises 200 inhabitants with 60 heads of households. Most of them are working in agriculture sector and also remain strongly dependent on homegardens and forest resources as source of food, income and medicine, owing to the vast traditional knowledge among them. Semi-structured interviews and field surveys were used to gather information on traditional knowledge of plants regarding the utilization of plant species, their usefulness and the utilized part. All of the head family units who managed the homegardens were interviewed, except the households that did not agree to participate. Homegardens were classified into three categories depending upon the size of homegarden holding: large, medium and small. Forty key informants representing 40 homegardens, 24 men and 16 women with ages ranging from 28 to 84 years were interviewed and the research background and purpose were explained prior to each interview. Total species and number of individuals of each useful plant species were also recorded. Plant pictures were taken during the field observation. Shannon-Wiener index (H) (Shannon and Weaver 1949) was used to calculate the species diversity. Principal component analysis (PCA) was used to investigate the relationship between the sizes of homegardens to the species richness by using the Past Software. Details on the traditional knowledge of plant species were summarized in Table 1.

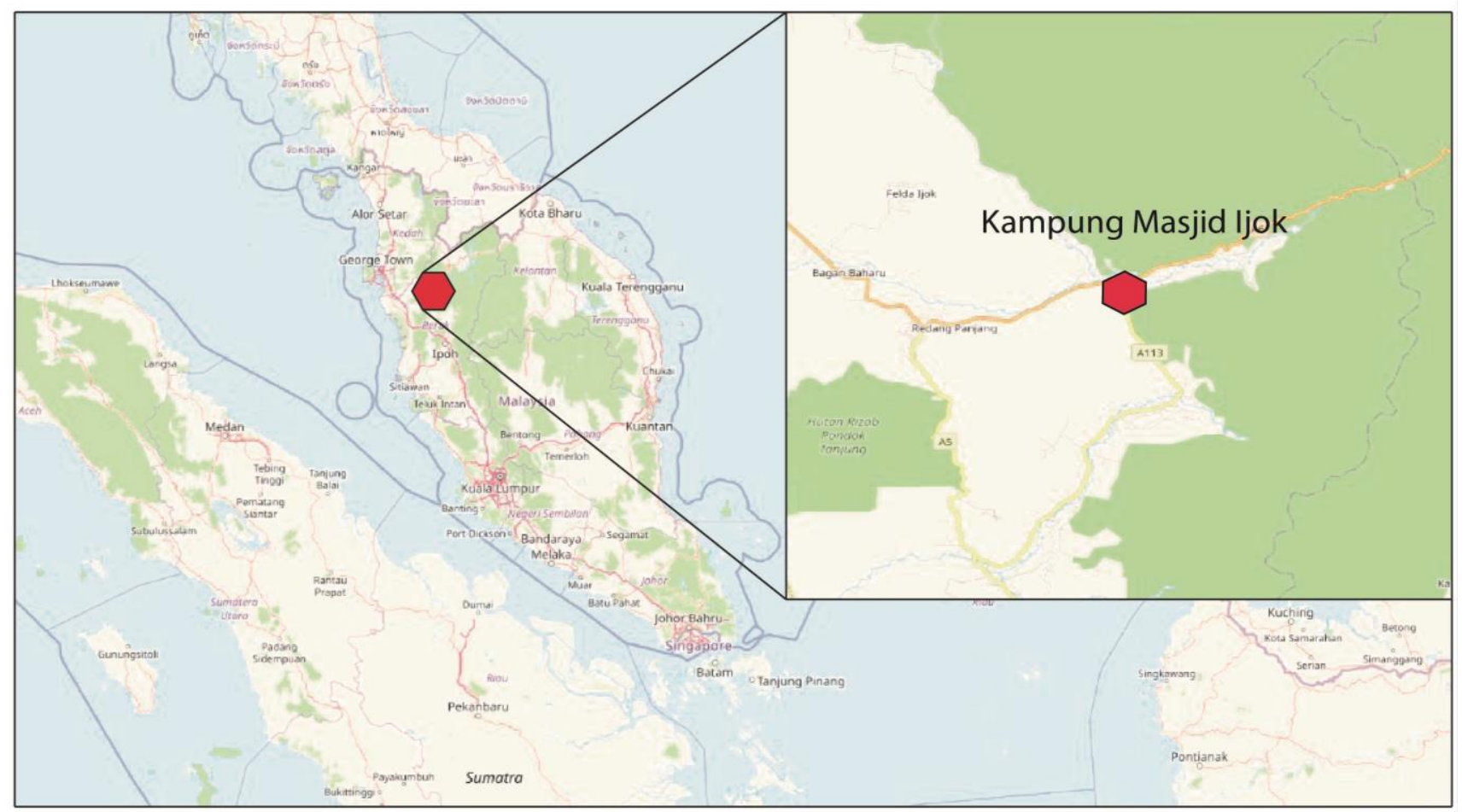

Figure 1. Location of Kampung Masjid Ijok, Perak, Malaysia lies between 507'0"N to 5 $5^{\circ} 15^{\prime \prime}$ latitude and $100^{\circ} 49^{\prime} 15^{\prime \prime}$ E to $100^{\circ} 50^{\prime} 45^{\prime \prime} \mathrm{E}$ longitude 


\section{RESULTS AND DISCUSSION}

Out of 40 studied homegardens, 9 were large $\left(>1,500 \mathrm{~m}^{2}\right), 11$ medium $\left(901 \mathrm{~m}^{2}\right.$ to $\left.1500 \mathrm{~m}^{2}\right)$ and $20 \mathrm{small}$ $\left(<900 \mathrm{~m}^{2}\right)$. Food, medicinal and ornamental plants dominated large homegardens, often with high multiple species, mainly used for family nutrition and commercialization, besides for medicinal and aesthetic values and largely represented by Durio zibethinus Murr, Garcinia mangostana L, Baccaurea motleyana Hook. f., Archidendron jiringa (Jack) I.C. Nielsen, Lansium domesticum Corrêa, Mangifera foetida Lour, Parkia speciosa Hassk, Pithecellobium bubalinum (Jack) Benth, Areca catechu L., Scorodocarpus borneensis (Baill.) Becc, Barringtonia racemosa (L)Spreng, Garcinia cambogia (Gaertn.) Desr and Musa paradisiaca L. Medium homegardens were dominated by fruit and medicinal plants, mainly used for complementing the family nutrition and wellbeing, and largely represented by Pandanus amaryllifolius Roxb, Languas galanga (L.) Stuntz, Piper sarmentosum Roxb. Manihot esculenta Crantz, Cassia alata L, Ananas comosus (L.) Merr, Capsicum frutescens L, Cymbopogon citratus (DC.) Stapf and Citrus aurantiifolia (Christm.) Swingle). Small homegardens consisted of many species of ornamental plants and few species of food and medicinal plants. The plants were mostly found in front yards. Examples of the plants were Hibiscus rosa-sinensis L, Ixora javanica (Blume) DC, Bougainvillea formosa W.Bull, Sansevieria trifasciata Prain, Codiaeum variegatum (L.) A. Juss, Heliconia psittacorum L.f, Euphorbia neriifolia L, Allamanda cathartica L, and Ruellia tweediana Griseb).

Though no specific planting pattern was observed, it was found that herbs and small shrub and ornamental plants were preferred in the front yard, while the boundaries and backyard consisted of shrubs and trees. The homegardens in the study area showed five distinct layers. The emergent layer was composed of big trees $(>20$ meters height), canopy layer middle size trees (between 10 to 20 meters height), understory layer fruit trees (between 5 to 10 meters height), shrub layer shrub (between 1 to 5 meters height) and ground layer herb, seedlings of both tree and shrub species. The common tree species in the emergent layer were Durio zibethinus Murr. and Melia excelsa Jack. Canopy layer was composed of Archidendron jiringa (Jack) I.C.Nielsen, Areca catechu L, Lansium domesticum Corrêa, Parkia speciosa Hassk and Scorodocarpus borneensis (Baill.) Becc. Understory layer was composed of Garcinia mangostana L, Baccaurea motleyana Hook. f., Mangifera foetida Lour, Garcinia cambogia (Gaertn.) and Barringtonia racemosa (L) Spreng. The shrub layer was composed of Citrus aurantiifolia (Christm.) Swingle, Etlingera elatior (Jack) R.M.Sm, Murraya koenigii (L.) Spreng and Bougainvillea formosa W.Bull. The ground layer was composed of Acanthus ebracteatus Vahl, Andrographis paniculata (Burm.f.) Wall. ex Nees, Amaranthus gangeticus L and Allium tuberosum Rottler ex Spreng.

A total of 207 species of useful plants distributed among 169 genera and 78 families were recorded from 40 homegardens in Kampung Masjid Ijok. The observation indicates the high biodiversity of plant species in a village of Peninsular Malaysia. The numbers of species found in this study are lower compared to other studies in a tropical region. Saikia et al. (2012) reported that there were294 plant species recorded in 80 homegardens from 17 villages of Upper Assam, India. Blanckaert et al. (2004) recorded 233 different plant species in 30 homegardens from a village of San Rafael Coxcatlan, Mexico. Bhat et al. (2014) reported 210 different plant species in 50 homegardens from 10 villages of Karwar, India. Although the total species is slightly lower than that found in other tropical regions, it is quite impressive given that this study was recorded for homegardens in a single village rather than a few villages.

Table 1. Floristic information of 40 homegardens

\begin{tabular}{llll}
\hline Scientific name & Family & Habit/life form & Use \\
\hline Abelmoschus esculentus (L.) Moench & Malvaceae & Shrub & Food \\
Acalypha hispida Burm.f. & Euphorbiaceae & Shrub & Ornamental \\
Acanthus ebracteatus Vahl & Acanthaceae & Herb & Medicinal \\
Agave americana L. & Agavaceae & Shrub & Ornamental \\
Ageratum conyzoides L. & Asteraceae & Herb & Medicinal \\
Allamanda cathartica L. & Apocynaceae & Shrub & Ornamental \\
Allium cepa L. & Amaryllidaceae & Herb & Food \\
Allium tuberosum Rottler ex Spreng. & Amaryllidaceae & Herb & Food \\
Alocasia denudata Engl. & Araceae & Shrub & Food \\
Alocasia macrorrhiza (L.) G.Don & Araceae & Shrub & Ornamental \\
Alocasia macrorrhiza Black Stem & Araceae & Shrub & Food \\
Alocasia sanderiana (W.Bull) Engl. & Araceae & Herb & Ornamental \\
Aloe vera (L.) Burm.f. & Xanthorrhoeaceae & Shrub & Ornamental \\
Alpinia officinarum Hance & Zingiberaceae & Herb & Medicinal \\
Amaranth gangeticus L. & Amaranthaceae & Herb & Food \\
Anacardium occidentale L. & Anacardiaceae & Tree & Food \\
\hline
\end{tabular}




\begin{tabular}{|c|c|c|c|}
\hline Ananas comosus (L.) Merr. & Bromeliaceae & Shrub & Food \\
\hline Ananas nanus L.B.Sm. & Bromeliaceae & Shrub & Medicinal \\
\hline Andrographis paniculata (Burm.f.) Wall. ex Nees & Acanthaceae & Herb & Medicinal \\
\hline Annona muricata $\mathrm{L}$. & Annonaceae & Tree & Medicinal \\
\hline Aquilaria malaccensis Benth. & Thymelaeaceae & Tre & Miscellaneous \\
\hline Archidendron jiringa (Jack)I.C.Nielsen & Fabaceae & Tree & Food \\
\hline Ardisia crenata Sims. & Primulaceae & Shrub & Food \\
\hline Areca catechu $\mathrm{L}$. & Arecaceae & Tree & Miscellaneous \\
\hline Artocarpus altilis (Parkinson) Fosberg & Moraceae & Tree & Food \\
\hline Artocarpus heterophyllus Lam. & Moraceae & Tree & Food \\
\hline Artocarpus integer (Thunb.) Merr. & Moraceae & Tree & Food \\
\hline Arundina graminifolia (D.Don) Hochr. & Orchidaceae & Shrub & Ornamental \\
\hline Averrhoa bilimbi L. & Oxalidaceae & Tree & Food \\
\hline Averrhoa carambola $\mathrm{L}$. & Oxalidaceae & Tree & Food \\
\hline Baccaurea macrocarpa Mull“.Arg. & Phyllanthaceae & Tree & Food \\
\hline Baccaurea motleyana Hook. f. & Phyllanthaceae & Tree & Food \\
\hline Barringtonia racemosa $(\mathrm{L})$ Spreng & Lecythidaceae & Tree & Food \\
\hline Begonia venosa Skan ex Hook.f & Begoniaceae & Herb & Ornamental \\
\hline Blumea balsamifera (L.) DC. & Asteraceae & Shrub & Medicinal \\
\hline Bouea macrophylla Griff. & Anacardiaceae & Tree & Food \\
\hline Bougainvillea formosa W.Bull & Nyctaginaceae & Shrub & Ornamental \\
\hline Caladium bicolour (Aiton) Vent. & Araceae & Herb & Ornamental \\
\hline Caladium humboldtii Vent. & Araceae & Herb & Ornamental \\
\hline Caladium lindenii (Andre)' Madison & Araceae & Herb & Ornamental \\
\hline Calamus manan Miq. & Arecaceae & Tree & Ornamental \\
\hline Calathea metallica Planch. \& Linden & Marantaceae & Shrub & Ornamental \\
\hline Calathea zebrina (Sims) Lindl. & Marantaceae & Shrub & Ornamental \\
\hline Capsicum frutescens $\mathrm{L}$. & Solanaceae & Climber & Medicinal \\
\hline Carica papaya $\mathrm{L}$ & Caricaceae & Shrub & Food \\
\hline Cassia alata $\mathrm{L}$ & Fabaceae & Shrub & Medicinal \\
\hline Catharanthus roseus (L.) G. Don & Apocynaceae & Shrub & Ornamental \\
\hline Ceiba pentandra (L.) Gaertn. & Malvaceae & Tree & Miscellaneous \\
\hline Chassalia curviflora (Wall.) Thwaites & Rubiaceae & Shrub & Medicinal \\
\hline Chrysothemis pulchella (Donn ex Sims) Decne. & Gesneriaceae & Herb & Ornamental \\
\hline Cinnamomum iners Reinw. ex Bl. & Lauraceae & Tree & Medicinal \\
\hline Citrus aurantiifolia (Christm.) Swingle & Rutaceae & Tree & Food \\
\hline Citrus hystrix DC. & Rutaceae & Tree & Food \\
\hline Citrus maxima (Burm. f.) Merr. & Rutaceae & Tree & Food \\
\hline Citrus medica (Christm. \& Panzer) Swingle & Rutaceae & Tree & Food \\
\hline Citrus suhuiensis Hayata & Rutaceae & Tree & Food \\
\hline Citrus $x$ microcarpa & Rutaceae & Tree & Food \\
\hline Claoxylon longifolium (Blume) Endl. Sx Hassk & Euphorbiaceae & Shrub & Food \\
\hline Clerodendron paniculatum $L$ & Verbenaceae & Shrub & Medicinal \\
\hline Clidemia hirta (L.) D. Don & Melastomataceae & Shrub & Medicinal \\
\hline Clinacanthus nutans(Burm. fil.) Linda & Acanthaceae & Shrub & Medicinal \\
\hline Cocos nucifera $\mathrm{L}$ & Arecacea & Tree & Food \\
\hline Codiaeum variegaantum (L.) A. Juss. & Euphorbiaceae & Shrub & Ornamental \\
\hline Coffea canephora Pierre ex A.Froehner & Rubiaceae & Shrub & Food \\
\hline Coleus blumei Benth. & Lamiaceae & Herb & Medicinal \\
\hline Colocasia esculenta (L.) Schott. & Araceae & Shrub & Food \\
\hline Colocasia gigantean (Blume ex Hassk.) Hook.f. & Araceae & Shrub & Food \\
\hline Cordyline terminalis Linn.Kunth & Agavaceae & Shrub & Ornamental \\
\hline Cosmos caudatus Kunth & Asteraceae & Herb & Food \\
\hline Costus speciosus (J.Konig) C.Specht & Costaceae & Shrub & Ornamental \\
\hline Costus spiralis (Jacq.) Roscoe. & Costaceae & Shrub & Ornamental \\
\hline Crinum asiaticum $\mathrm{L}$. & Amaryllidaceae & Shrub & Ornamental \\
\hline Crinum pedunculatum $\mathrm{R} . \mathrm{Br}$. & Amaryllidaceae & Shrub & Ornamental \\
\hline Cucumis sativus $\mathrm{L}$. & Cucurbitaceae & Climber & Food \\
\hline Cucurbita moschata Duchesne ex Poir. & Cucurbitaceae & Climber & Food \\
\hline Curcuma domestica Valeton & Zingiberaceae & Herb & Food \\
\hline Cycas macrocarpa Griff. & Cycadaceae & Shrub & Ornamental \\
\hline Cymbopogon citratus (DC.) Stapf & Poaceae & Shrub & Food \\
\hline Cymbopogon nardus (L.) Rendle & Poaceae & Shrub & Medicinal \\
\hline Cynometra cauliflora $\mathrm{L}$. & Fabaceae & Tree & Food \\
\hline Cyperus alternifolius $\mathrm{L}$ & Cyperaceae & Shrub & Medicinal \\
\hline Dieffenbachia maculate (Lodd.) G.Don. & Araceae & Shrub & Ornamental \\
\hline Dillenia suffruticosa (Griff.) Martelli. & Dilleniaceae & Tree & Ornamental \\
\hline
\end{tabular}




\begin{tabular}{|c|c|c|c|}
\hline Dimocarpus longan Lour & Sapindaceae & Tree & Food \\
\hline Durio zibethinus Murr & Malvaceae & Tree & Food \\
\hline Dypsis lutescens (H.Wendl.) Beentje \& J.Dransf. & Arecacea & Tree & Ornamental \\
\hline Eichhornia crassipes (Mart.) Solms & Pontederiaceae & Herb & Ornamental \\
\hline Elaeis guineensis Jacq. & Arecaceae & Tree & Miscellaneous \\
\hline Episcia cupreata (Hook.) Hanst. & Lamiales & Herb & Ornamental \\
\hline Ervatamia coronaria (Jacq.) Stapf & Apocynaceae & Shrub & Ornamental \\
\hline Etlingera coccinea (Blume) S.Sakai \& Nagam. & Zingiberaceae & Herb & Medicinal \\
\hline Etlingera elatior (Jack) R.M.Sm. & Zingiberaceae & Shrub & Food \\
\hline Etlingera megalocheilos (Griff.) A.D.Poulsen & Zingiberaceae & Shrub & Food \\
\hline Eugenia aquea Burm. f. & Myrtaceae & Tree & Food \\
\hline Euphorbia hirta L. & Euphorbiaceae & Herb & Medicinal \\
\hline Euphorbia mili Des Moul. & Euphorbiaceae & Shrub & Ornamental \\
\hline Euphorbia neriifolia L. & Euphorbiaceae & Shrub & Ornamental \\
\hline Eurycoma longifolia Jack & Simaroubaceae & Tree & Food \\
\hline Ficus schwarzii Koord & Moraceae & Tree & Food \\
\hline Garcinia cambogia (Gaertn.) Desr. & Clusiaceae & Tree & Food \\
\hline Garcinia mangostana $\mathrm{L}$ & Clusiaceae & Tree & Food \\
\hline Gigantochloa albociliata (Munro) Kurz & Poaceae & Tree & Food \\
\hline Gomphrena globosa L. & Amaranthaceae & Herb & Ornamental \\
\hline Gynura sarmentosa (Blume) DC. & Compositae & Herb & Medicinal \\
\hline Heliconia bicolor Benth. & Heliconiaceae & Shrub & Ornamental \\
\hline Heliconia psittacorum L.f. & Heliconiaceae & Shrub & Ornamental \\
\hline Hevea brasiliensis (Willd. ex A.Juss.) Mull".Arg & Euphorbiaceae & Tree & Miscellaneous \\
\hline Hibiscus rosa-sinensis $\mathrm{L}$. & Malvaceae & Shrub & Ornamental \\
\hline Hibiscus sabdariffa L. & Malvaceae & Shrub & Ornamental \\
\hline Huperzia carinata Desv. Ex Poir) Trevisan & Huperziaceae & Herb & Ornamental \\
\hline Hylocereus undatus (Haw.) Britton \& Rose & Cactaceae & Shrub & Food \\
\hline Impatiens balsamina $\mathrm{L}$. & Balsaminaceae & Herb & Ornamental \\
\hline Ipomoea aquatica Forssk. & Convolvulaceae & Herb & Food \\
\hline Ipomoea batatas (L.) Lam. & Convolvulaceae & Herb & Food \\
\hline Ixora javanica (Blume) DC. & Rubiaceae & Shrub & Ornamental \\
\hline Kalanchoe pinnata (Lam.) Pers. & Crassulaceae & Herb & Medicinal \\
\hline Languas galanga (L.) Stuntz & Zingiberaceae & Shrub & Food \\
\hline Lansium domesticum Correa & Meliaceae & Tree & Food \\
\hline Laurentia longiflora (Linn) Paterm & Campanulaceae & Herb & Medicinal \\
\hline Lawsonia inermis L. & Lythraceae & Shrub & Miscellaneous \\
\hline Lepisanthes rubiginosa (Roxb.) Leenh & Sapindaceae & Tree & Food \\
\hline Leucaena leucocephala (Lamk) De Wit & Fabaceae & Tree & Medicinal \\
\hline Licuala peltata Roxb. ex Buch. & Arecacea & Shrub & Ornamental \\
\hline Licuala spinosa Roxb. & Arecaceae & Shrub & Miscellaneous \\
\hline Limnocharis flava (L.) Buchenau & Alismataceae & Herb & Food \\
\hline Lycopodium phlegmaria A. Cunn. & Huperziaceae & Herb & Ornamental \\
\hline Mallotus barbatus Mull".Arg. & Euphorbiaceae & Tree & Medicinal \\
\hline Mangifera foetida Lour. & Anacardiaceae & Tree & Food \\
\hline Mangifera indica $\mathrm{L}$. & Anacardiaceae & Tree & Food \\
\hline Manihot esculenta Crantz & Euphorbiaceae & Shrub & Food \\
\hline Manilkara zapota (L.) P.Royen & Sapotaceae & Tree & Food \\
\hline Melastoma decemfidum Roxb. & Melastomataceae & Shrub & Medicinal \\
\hline Melia excelsa Jack & Meliaceae & Tre & Food \\
\hline Metroxylon sagu Rottb. & Arecacea & Tree & Food \\
\hline Mimosa pudica L. & Fabaceae & Herb & Medicinal \\
\hline Mirabilis longiflora $\mathrm{L}$. & Nyctaginaceae & Herb & Ornamental \\
\hline Molineria latifolia (Dryand. ex W.T.Aiton) Herb. ex Kurz & Hypoxidaceae & Herb & Medicinal \\
\hline Momordica charantia $\mathrm{L}$ & Cucurbitaceae & Climber & Food \\
\hline Morinda citrifolia $\mathrm{L}$. & Rubiaceae & Tree & Medicinal \\
\hline Murraya koenigii (L.) Spreng. & Rutaceae & Tree & Food \\
\hline Musa paradisiaca $\mathrm{L}$. & Musaceae & Shrub & Miscellaneous \\
\hline Nepenthes gracilis Korth. & Nepenthaceae & Herb & Ornamental \\
\hline Nephelium lappaceum L. & Sapindaceae & Tree & Food \\
\hline Nephelium mutabile Bl. & Sapindaceae & Tree & Food \\
\hline Ocimum gratissimum Forssk. & Labiatae & Shrub & Medicinal \\
\hline Oenanthe javanica (Blume) DC. & Apiaceae & Herb & Food \\
\hline Oroxylum indicum (L.) Kurz & Bignoniaceae & Tree & Medicinal \\
\hline Orthosiphon aristatus (Blume) Miq. & Labiatae & Shrub & Medicinal \\
\hline Oxalis barrelieri L. & Oxalidaceae & Herb & Medicinal \\
\hline Oxalis triangularis A.St.-Hil. & Oxalidaceae & Herb & Ornamental \\
\hline
\end{tabular}




\begin{tabular}{|c|c|c|c|}
\hline Pachyrhizus erosus (L.) Urb. & Fabaceae & Climber & Food \\
\hline Pandanus amaryllifolius Roxb. & Pandanaceae & Shrub & Food \\
\hline Pandanus caricosus Spreng. & Pandanaceae & Shrub & Miscellaneous \\
\hline Parkia speciosa Hassk. & Fabaceae & Tree & Food \\
\hline Passiflora edulis Sims & Passifloraceae & Climber & Food \\
\hline Pedilanthus tithymaloides (L.) Poit. & Euphorbiaceae & Shrub & Ornamental \\
\hline Peperomia pellucida Kunth & Piperaceae & Herb & Food \\
\hline Persicaria hydropiper L. & Polygonaceae & Herb & Food \\
\hline Phyllanthus niruri L & Phyllanthaceae & Herb & Medicinal \\
\hline Phyllanthus pulcher Wall.ex Mull.Arg. & Euphorbiaceae & Shrub & Medicinal \\
\hline Phyllanthus pulcher Wall.ex Mull.Arg. & Euphorbiaceae & Shrub & Medicinal \\
\hline Physalis minima $\mathrm{L}$ & Solanaceae & Climber & Medicinal \\
\hline Physalis minima $L$ & Solanaceae & Climber & Medicinal \\
\hline Piper betle L. & Piperaceae & Climber & Medicinal \\
\hline Piper sarmentosum Roxb. & Piperaceae & Herb & Food \\
\hline Pithecellobium bubalinum (Jack) Benth. & Fabaceae & Tree & Food \\
\hline Platycerium coronarium (D. Konig“" ex O.F. Mull“.) Desv. & Polypodiaceae & Shrub & Ornamental \\
\hline Plumeria rubra $\mathrm{L}$. & Apocynaceae & Tree & Ornamental \\
\hline Portulaca grandiflora Hook. & Portulacaceae & Herb & Ornamental \\
\hline Psidium guajava $\mathrm{L}$. & Myrtaceae & Tree & Food \\
\hline Psophocarpus tetragonolobus (L.) D.C & Fabaceae & Climber & Food \\
\hline Pteris ensiformis Burm. & Pteridaceae & Herb & Ornamental \\
\hline Punica granatum $\mathrm{L}$. & Lythraceae & Shrub & Food \\
\hline Pyrrosia piloselloides (L.) M.G.Price & Polypodiaceae & Herb & Medicinal \\
\hline Rhinacanthus communis Nees & Acanthaceae & Herb & Medicinal \\
\hline Ricinus communis L. & Euphorbiaceae & Shrub & Medicinal \\
\hline Ricinus communis $\mathrm{L}$. & Euphorbiaceae & Shrub & Medicinal \\
\hline Rosa L. & Rosaceae & Shrub & Ornamental \\
\hline Ruellia tweediana Griseb. & Acanthaceae & Shrub & Ornamental \\
\hline Saccharum officinarum L. & Poaceae & Shrub & Food \\
\hline Salacca zalacca (Gaertn.) Voss. & Arecaceae & Shrub & Food \\
\hline Sansevieria cylindrical Bojer ex Hook. & Asparagaceae & Shrub & Ornamental \\
\hline Sansevieria trifasciata Prain & Asparagaceae & Shrub & Ornamental \\
\hline Sauropus androgynus (L.) Merr. & Euphorbiaceae & Shrub & Food \\
\hline Sauropus androgynus (L.) Merr. & Euphorbiaceae & Shrub & Food \\
\hline Scorodocarpus borneensis (Baill.) Becc. & Olacaceae & Tree & Medicinal \\
\hline Sida rhombifolia $\mathrm{L}$. & Malvaceae & Herb & Medicinal \\
\hline Smilax myosotiflora A.DC. & Smilacacaceae & Tree & Medicinal \\
\hline Solanum ferox $\mathrm{L}$. & Solanaceae & Shrub & Food \\
\hline Solanum ferox $L$ & Solanaceae & Shrub & Food \\
\hline Solanum lasiocarpum Dunal & Solanaceae & Shrub & Food \\
\hline Solanum lasiocarpum Dunal & Solanaceae & Shrub & Food \\
\hline Solanum melongena $\mathrm{L}$. & Solanaceae & Shrub & Food \\
\hline Solanum melongena $L$. & Solanaceae & Shrub & Food \\
\hline Solanum torvum Sw. & Solanaceae & Shrub & Medicinal \\
\hline Solanum torvum $S w$. & Solanaceae & Shrub & Medicinal \\
\hline Spathoglottis plicata Blume & Orchidaceae & Shrub & Ornamental \\
\hline Spondias pinnata (L. f.) Kurz & Anacardiaceae & Tree & Food \\
\hline Stachytarpheta jamaicensis (L.) Vahl & Verbenaceae & Shrub & Medicinal \\
\hline Syzygium jambos L. (Alston) & Myrtaceae & Tree & Food \\
\hline Syzygium malaccense (L.) Merr. \& L.M.Perry & Myrtaceae & Tree & Food \\
\hline Tacca integrifolia Ker Gawl. & Dioscoreaceae & Herb & Medicinal \\
\hline Talinum paniculatum (Jacq.) Gaertn. & Portulacaceae & Shrub & Medicinal \\
\hline Theobroma cacao L. & Malvaceae & Tree & Food \\
\hline Tunera ulmifolia $L$. & Turneraceae & Shrub & Ornamental \\
\hline Urena lobata $\mathrm{L}$ & Malvaceae & Shrub & Ornamental \\
\hline Vernonia amygdalina Delile & Asteraceae & Shrub & Medicinal \\
\hline Vernonia cinera (Linn). Less. & Asteraceae & Herb & Medicinal \\
\hline Vigna sesquipedalis (L.)Fruwirth & Fabaceae & Climber & Food \\
\hline Vitex pubescens Vahl. & Verbenaceae & Tree & Medicinal \\
\hline Wedelia trilobata (L.) A.S. Hitchc. & Asteraceae & Herb & Ornamental \\
\hline Xanthosoma sagittifolium (L.) Schott. & Araceae & Shrub & Food \\
\hline Zea mays $\mathrm{L}$. & Poaceae & Shrub & Food \\
\hline Zingiber efficinale Roscoe & Zingiberaceae & Shrub & Food \\
\hline Zingiber zerumbet (L.) Smith & Zingiberaceae & Shrub & Medicinal \\
\hline
\end{tabular}


In this study, the most frequent species recorded (Musa paradisiaca L., Cocos nucifera L., Nephelium lappaceum L., Capsicum frutescens L., Carica papaya L, Cymbopogon citratus (DC.) Stapf, Mangifera indica L, Curcuma domestica Valeton, Garcinia mangostana L, Manihot esculenta Crantz, Lansium domesticum Correa, Areca catechu L.) are consistent with literature reported on homegardens in tropical region. Caballero-Serrano et al. (2016) reported that the most common species documented in homegardens of Ecuador were Musa spp., Colocasia esculenta, Manihot esculenta, Mouriri spp. and Ananas comosus. In Tuyen Quang, Vietnam, Musa spp., Zingiber officinale and Colocasia esculenta were among the most common plants recorded in homegardens (Timsuksai et al. 2015). In Karen, Thailand, Panyadee et al. (2018) concluded that the most common species documented in homegardens were Mangifera indica, Artocarpus heterophyllus, Psidium guajava, and Colocasia esculenta.

The common occurrence of plant species in this study with the other homegardens in tropical regions was probably due to similar climatic conditions, but the differences in their frequencies and abundance could be due to the variation in the social-cultural characteristics and biophysical condition. For example, the high frequencies of Capsicum frutescens L, Cymbopogon citratus (DC.) Stapf and Curcuma domestica Valeton in this study could be due primarily to its usage as a main ingredient in most Malay traditional cuisine. Cymbopogon citratus (DC.) Stapf was also recorded in homegardens of Paraíba, Northeastern Brazil (Carvalho et al. 2013) and Mayan homegardens, Mexico (de Clerck and Negreros-Castillo 2000) but Curcuma domestica Valeton which was highly recorded in this study was not found in their homegardens. At the family level, Euphorbiaceae (13 species), Araceae (11 species), Fabaceae (10 species), Arecaceae (9 species), Malvaceae (8 species), Zingiberaceae (8 species), and Rutaceae ( 7 species) demonstrated the highest species composition of useful plants in homegardens. In terms of habit, shrubs dominated the plants in all homegardens with a portion of $42 \%$, followed by trees (29.5\%), herbs $(23.7 \%)$ and climbers $(4.8 \%)$ as shown in Figure 2. As many as $43.3 \%$ of the useful plant species were exclusively used as food. $27.8 \%$ and $24 \%$ of the species as ornamental and medicine. The remaining $4.9 \%$ of the plant species had other uses, and were grouped under the category miscellaneous. Figure 3 shows the usage of plants in the homegardens.

According to Fernandes and Nair (1986) homegardens are an important source of subsistence products due to unavailability of the products in the market, leading to higher species diversity in remote villages. In this study, the highest frequency of Musa Paradisiaca indicates the importance of this plant for household consumption and the economy as the excess production of the product is sold to increase their household income. Fruit plant species in particular, dominated the biodiversity found in the homegardens, accounting for $43.3 \%$ of the total species. The ten most frequently found species in homegardens also fall under this category. A similar observation was also reported by George and Christopher (2020) in Kerala, India, Sujarwo and Caneva (2015) in Bali, Indonesia, Srithi et al. (2012) in Thailand and Roy et al. (2013) in Bangladesh. It has been reported that fruit plants make an important contribution to the nutrition of households (Nair 1993). A few species of useful plants are recorded in the homegardens that are observed to be found in the forests surrounding the homegardens, i.e., Scorodocarpus borneensis (Baill) Becc and Eurycoma longifolia Jack, which are used for medicinal purposes by the local community. Scorodocarpus borneensis (Baill) Becc has been traditionally used by the local community in Kampung Masjid Ijok to treat indigestion problems, while Eurycoma longifolia Jack is used to treat low sexual energy. According to Lim (2012), Scorodocarpus borneensis (Baill) Becc is found in undisturbed to slightly disturbed (open) mixed dipterocarp forests up to $900 \mathrm{~m}$ altitudes, while Eurycoma longifoliaJack is a slender, evergreen flowering tree, occurring naturally in jungles of Malaysia and Indonesia (Wernsdorfer et al. 2009). Besides medicinal purposes, some of the plants are also domesticated for their fruit. Such plants are Parkia speciosa Hassk which has been reported to be distributed in the Malaysian forests (Huey et al. 2014) and Garnia cambogia (Gaertn), naturally found in the lowland tropical rainforest (Lim 2012). Homegardens at the study site also contribute to the conservation of plant species. There were two species of plants categorized as critically endangered (CR) in (IUCN) Red List namely Aquilaria malaccensis and Alocasia sanderiana.

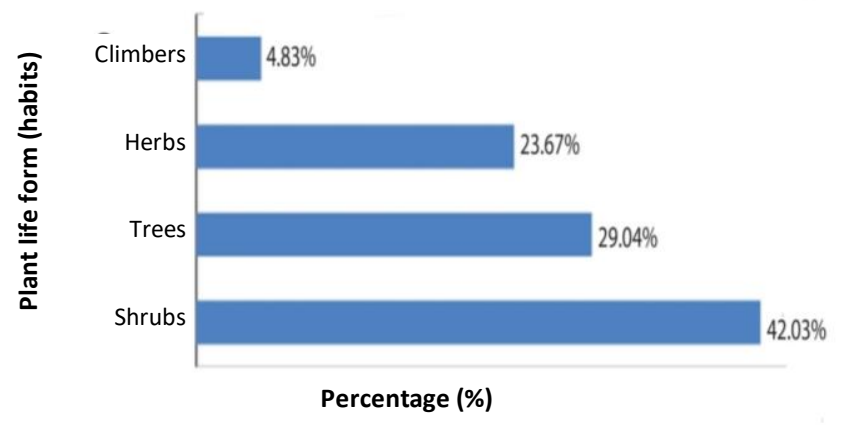

Figure 2. Diversity of life forms (habits) of plants in homegardens

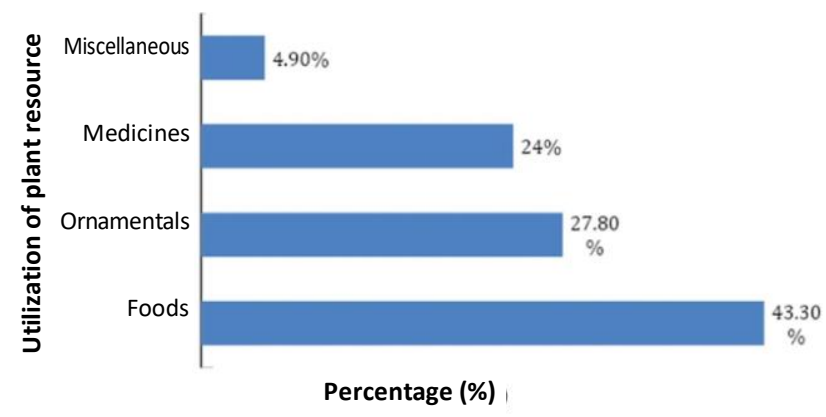

Figure 3. Uses of homegarden plants 
According to Kumar and Nair (2004) the diversity of plants in homegardens is closely related to how the homegardens are being managed. The integration of multiple plant species with different life forms (tree, shrub, herb, climbers) has created proper dynamics in homegardens. It has been reported in the literature, most homegardens are made up of plants in multiple life-forms, but slightly different in composition and dominance. Sujarwo and Caneva (2015) reported that tree-dominated homegardens of Bali, Indonesia, followed by shrub, herb, and climber. In homegardens of Ethiopia, Mekonen et al. (2015) reported that herb dominated, followed by tree, shrub and climber, while a study by Barbhuiya et al. (2016) reported that tree dominated homegardens of Northeastern India, followed by shrub and herbs. The interaction between plants with multiple life-forms provides benefits to the environment. It has been reported that trees could dry and aerate soils by intercepting rainfall and transferring water from the soil to atmosphere through transpiration, increase soil nutrient availability by litterfall and reduce irradiance by shading (Holmgren et al. 2015). Molder et al. (2008) reported a significant correlation between tree-herb layers and environmental parameters like $\mathrm{pH}$, light, and humus thickness. In this study, the tree constituted $29.47 \%$ of the total species recorded. The average trees recorded were between 6 to 20 species per homegardens. A nitrogenfixating tree in this study, namely Leucaena leucocephala (Lam.) de Wit., can enrich the soil with nitrogen (Liu et al.
2018). The highest Shannon-Weiner Index was recorded in the large-sized homegardens (3.61) and the lowest in smallsized homegardens (1.66). Principle Component Analysis (PCA) incorporating several variables (size of homegardens, total species and Shannon-Weiner index) indicated that large homegardens corresponds to the high total number of species and Shannon-Weiner index (Figure 4). This study suggested that large homegardens are more effective for the conservation of plant diversity than small and medium ones. This result is consistent with the findings by Mekonen et al. (2015), who noted that diversity of plant species is correlated with the size of homegardens.

To conclude, this current floristic survey of 40 homegardens in the Kampung Masjid Ijok, Perak, Malaysia reveals that many food plants, ornamental and medicinal plants are still widely found and used by the community. The high diversity of plant species recorded in this study provides a lot of benefits to the environment and the economy and social of the local community. Alocasia sanderiana and Aquilaria malaccensis categorized as critically endangered (CR) in (IUCN) Red List were also recorded in the study site. These findings indicate that homegardens are suitable places for conservation of these plants. Given the significant contribution and benefits of home gardens to human well-being and the lack of exploration of home gardens in Malaysia, this study could provide useful information that can support and encourage local communities to develop their home gardens in a sustainable manner.

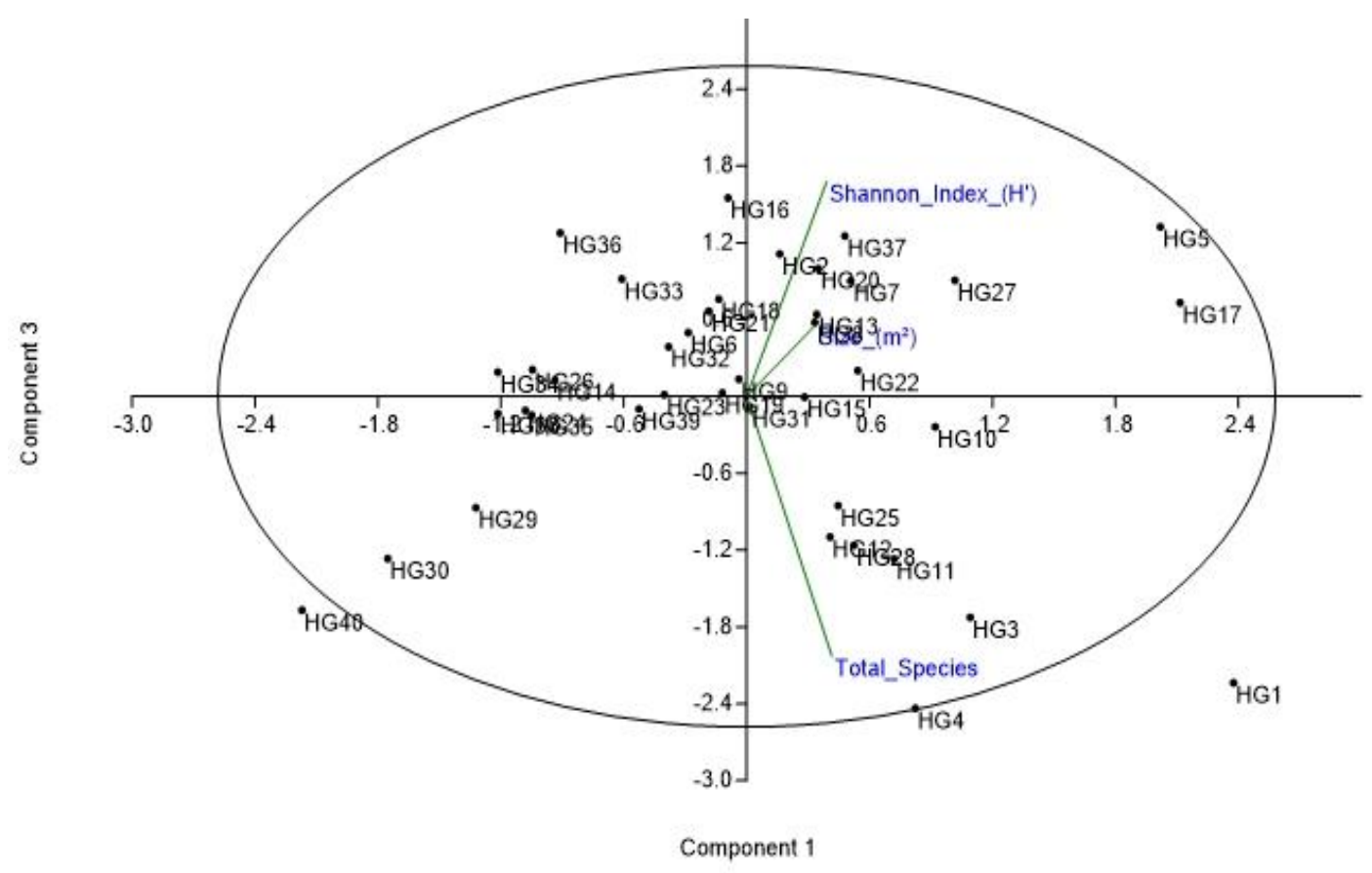

Figure 4. PCA indicates correlation between size of homegardens, total species and Shannon-Wiener index 


\section{ACKNOWLEDGEMENTS}

We thank the University Malaya and the Ministry of Education of Malaysia for providing facilities and funds that enable this study to be carried out. Publication of this article was funded by PG072-2013A, RF193-12SUS and LL023-16SUS provided by University of Malaya and MyBrain 15 Scholarship provided by Ministry of Education of Malaysia. This study is also to be a part of a Ph.D. contribution of the first author.

\section{REFERENCES}

Abdoellah OS, Schneider M, Nugraha LM, Suparman Y, Voletta CT, Withaningsih S, Parikesit, Heptiyanggit A, Hakim L. 2020 Homegarden commercialization: extent, household characteristics, and effect on food security and food sovereignty in Rural Indonesia. Sustain Sci 15 (3): 797-815. DOI: 10.1007/s11625-020-00788-9.

Avilez-Lopez T, van der Wal H, Aldasoro-Maya EM, Rodriguez-Robles U. 2020. Home gardens' agrobiodiversity and owners' knowledge of their ecological, economic and socio-cultural multifunctionality: a case study in the lowlands of Tabasco, México. J Ethnobiol Ethnomed 16 (42): 1-13. DOI: 10.1186/s13002-020-00392-2.

Barbhuiya AR, Sahoo UK, Upadhyaya K. 2016. Plant diversity in the indigenous home gardens in the Eastern Himalayan Region of Mizoram, Northeast India. Econ Bot 70 (2): 115-131. DOI: 10.1007/s12231-016-9349-8.

Bardhan S, Jose S, Biswas S, Kabir K, Rogers W. 2012. Homegarden agroforestry systems: an intermediary for biodiversity conservation in Bangladesh. Agrofor Syst 85 (1): 29-34. DOI: 10.1007/s10457-0129515-7.

Bargali SS, Padalia K, Bargali K. 2019. Effects of tree fostering on soil health and microbial biomass under different land-use systems in central Himalaya. Land Degrad Dev 30 (16): 1984-1998. DOI: 10.1002/ldr.3394.

Bhat S, Bhandary MJ, Rajanna L. 2014. Plant diversity in the homegardens of Karwar, Karnataka, India. Biodiversitas 15 (2): 229235. DOI: 10.13057/biodiv/d150216.

Blanckaert I, Swennen RL, Flores PM, Lopez RI, Saade RL. 2004. Floristic composition, plant uses and management practices in homegardens of San Rafael Coxcatlan, valley of Tehuacan-Cuicatlan, Mexico. J Arid Environ 57(2): 179-202. DOI:10.1016/S0140 1963(03)00100-9.

Caballero-Serrano V, Onaindia M, Alday JG, Caballero D, Carrasco JC, McLaren B, Amigo J. 2016. Plant diversity and ecosystem services in Amazonian homegardens of Ecuador. Agric Ecosyst Environ 225:116-125. DOI: 10.1016/j.agee.2016.04.005.

Carvalho TKN, de Oliveira A, de Lucena CM, Pedrosa KM, Neto CFADV, Alves CAB, Felix LP, Florentino ATNF, Alves RRDN, de Andrade LA, de Lucena RFP. 2013. Structure and floristics of home gardens in an altitudinal marsh in Northeastern Brazil. Ethnobot Res Appl 11: 29-48.

de Clerck FAJ, Negreros-Castillo P. 2000. Plant species of traditional Mayan homegardens of Mexico as analogs for multistrata agroforests. Agrofor Syst 48 (3): 303-317. DOI: 10.1023/A:1006322612362.

Ebert AW. 2020. The role of vegetable genetic resources in nutrition security and vegetable breeding. Forest 9 (736): 1-20. DOI: 10.3390/plants9060736.

Fernandes ECM, Nair PKR. 1986. An evaluation of the structure and function of tropical home gardens. Agric Syst 21 (4): 279-310. DOI: 10.1016/0308-521X(86)90104-6.

Gachuiri AN, Carsan S, Karanja E, Makui P, Nyaguthii A, Carsan S, Karanja E. 2017. Diversity and importance of local fodder tree and shrub resources in mixed farming systems of central Kenya. For Trees Livelihoods 26 (3): 143-155. DOI: 10.1080/14728028.2017.1316216.

Galhena DH, Freed R, Maredia KM. 2013. Home gardens: a promising approach to enhance household food security and wellbeing. Agric Food Secur 2 (8): 1-13. DOI: 10.1186/2048-7010-2-8.

George MV, Christopher G. 2020. Structure, diversity and utilization of plant species in tribal homegardens of Kerala, India. Agrofor Syst 94 (1): 297-307. DOI: 10.1007/s10457-019-00393-5.
Holmgren M, Lin CY, Murillo JE, Nieuwenhuis A, Penninkhof J, Sanders N, Bart TV, Veen HV, Vasander H, Vollebregt ME, Limens J. 2015. Positive shrub-tree interactions facilitate woody encroachment on boreal peatlands. J Ecol 103 (1): 58-66. DOI: 10.1111/13652745.12331 .

Huey JK, Lai HA, Lean TN. 2014. Antioxidant activities and polyphenolic constituents of bitter bean Parkia speciosa. Intl J Food Prop 17 (9): 1977-1986. DOI: 10.1080/10942912.2013.775152.

Kumar BM, Nair PR. 2004. The enigma of tropical homegardens. Agrofor Syst 61 (1): 135-152. DOI: 10.1023/B:AGFO.0000028995.13227.ca

Lautenbach S, Seppelt R, Liebscher J, Dormann CF. 2012. Spatial and temporal trends of global pollination benefit. PLoS ONE 7: e35954. DOI: 10.1371/journal.pone.0035954.

Lim TK. 2012. Edible Medicinal and Non-Medicinal Plants: Volume 4, Fruits. Springer Science Business Media B.V., New York.

Linger W. 2014. Agro-ecosystem and socio-economic role of homegarden agroforestry in Jabithenan District, North-Western Ethiopia: implication for climate change adaptation. SpringerPlus 3 (154): 1-9. DOI: 10.1186/2193-1801-3-154

Liu FY, Gao CJ, Chen M, Li L. 2018. Above- and below-ground biomass relationships of Leucaena leucocephala (Lam.) de Wit in different plant stands. PLoS ONE 13: e0207059. DOI: 10.1371/journal.pone.0207059.

Mapongmetsem PM, Nkongmeneck BA, Gubbuk H. 2012. Socioeconomic importance of the banana tree (Musa spp.) in the Guinean Highland Savannah agroforests. Sci World J 2012: 1-8. DOI: $10.1100 / 2012 / 350258$

Mattsson E, Ostwald M, Nissanka SP, Marambe B. 2013. Homegardens as a multi-functional land-use strategy in Sri Lanka with focus on carbon sequestration. Ambio 42 (7): 892-902. DOI: 10.1007/s13280-0130390-x

Mattsson E, Ostwald M, Nissanka SP, Pushpakumara DKNG. 2015. Quantification of carbon stock and tree diversity of homegardens in a dry zone area of Moneragala District, Sri Lanka. Agrofor Syst 89 (3): 435-445. DOI: 10.1007/s10457-014-9780-8.

Mattsson E, Ostwald M, Nissanka SP. 2017. What is good about Sri Lankan homegardens with regards to food security? A synthesis of the current scientific knowledge of a multifunctional land-use system. Agrofor Syst 92 (6): 1469-1484. DOI: 10.1007/s10457-017-0093-6.

Mekonen T, Giday M, Kelbessa E. 2015. Ethnobotanical study of homegarden plants in Sebeta582 Awas District of the Oromia Region of Ethiopia to assess use, species diversity and management practices. J Ethnobiol Ethnomed 11 (64): 1-13. DOI: 10.1186/s13002-0150049-8

Milow P, Malek S, Mohammad NS, Ong HC. 2013. Diversity of plants tended or cultivated in Orang Asli Homegardens in Negeri Sembilan, Peninsular Malaysia. Hum Ecol 41 (2): 325-331. DOI: 10.1007/s10745-012-9555-7.

Mohri H, Lahoti S, Saito O, Mahalingam A, Gunatilleke N, Van Thang Hoang I, Hitinayake G, Takeuchi K, Herath S. 2013. Assessment of ecosystem services in homegarden systems in Indonesia, Sri Lanka, and Vietnam. Ecosyst Serv 5: 124-136. DOI: 10.1016/j.ecoser.2013.07.006.

Molder A, Bernhardt-Römermann M, Schmidt W. 2008. Herb-layer diversity in deciduous forests: raised by tree richness or beaten by beech? For Ecol Manag 256 (3): 272-281. DOI: 10.1016/j.foreco.2008.04.012.

Nair PKR. 1993. An Introduction to Agroforestry. ICRAF \& Kluwer Academic Publishers, Nederland.

Pala NA, Sarkar BC, Shukla G, Chettri N, Deb S, Bhat JA, Chakravarty S. 2019. Floristic composition and utilization of ethnomedicinal plant species in home gardens of the Eastern Himalaya. J Ethnobiol Ethnomed 15 (14): 1-16. DOI: 10.1186/s13002-019-0293-4

Panyadee P, Balslev H, Wangpakapattanawong P, Inta A. 2018. Karen homegardens: Characteristics, functions, and species diversity. Econ Bot 72 (1): 1-19. DOI: 10.1007/s12231-018-9404-8

Panyadee P, Balslev H, Wangpakapattanawong P, Inta A. 2019. Medicinal plants in homegardens of four ethnic groups in Thailand. J $\begin{array}{llll}\text { Ethnopharmacol } 239 & \text { (111927): } & 1-14 . & \text { DOI: }\end{array}$ 10.1016/j.jep.2019.111927

Reetsch A, Schwärzel K, Dornack C, Stephene S, Feger KH. 2020. Optimising nutrient cycles to improve food security in smallholder farming families - A case study from banana-coffee-based farming in the Kagera Region, NW Tanzania. Sustainability 12 (9105): 1-34. DOI: $10.3390 /$ su12219105. 
Roy B, Rahman MH, Fardusi MJ. 2013. Status, diversity, and traditional uses of homestead gardens in Northern Bangladesh: A means of sustainable biodiversity conservation. ISRN Biodivers 124103: 1-11. DOI: $10.1155 / 2013 / 124103$.

Saikia P, Choudhury, BI, Khan AL. 2012. Floristic composition and plant utilization pattern in homegardens of Upper Assam, India. Trop Ecol 53 (1): 105-118.

Shannon CE, Weaver EW. 1949. The mathematical theory of communication. University of Illinois Press, Urbana, IL.

Srithi K, Trisonthi C, Wangpakapattanawong P, Srisanga P, Balslev H. 2012. Plant diversity in Hmong and Mien homegardens in Northern
Thailand. Econ Bot 66 (2): 192-206. DOI: 10.1007/s12231-012-9199y.

Sujarwo W, Caneva G. 2015. Ethnobotanical study of cultivated plants in home gardens of traditional villages in Bali (Indonesia). Hum Ecol 43 (5): 769-778. DOI: 10.1007/s10745-015-9775-8.

Timsuksai P, Tien ND, Rambo AT. 2015. Homegardens of the Cao Lan, a Tai-speaking ethnic minority in Vietnam's Northern Mountains. Southeast Asian Stud 4 (2): 365-383

Wernsdorfer WH, Ismail S, Chan KL, Congpuong K, Wernsdorfer G. 2009. Activity of Eurycoma longifolia root extract against Plasmodium falciparum in vitro. Wien Klin Wochenschr 121 (3): 2326. DOI: $10.1007 / \mathrm{s} 00508-009-1230-7$. 